\title{
Article \\ Asymptotic Behavior of Resolvents of a Convergent Sequence of Convex Functions on Complete Geodesic Spaces
}

\author{
Yasunori Kimura (D) and Keisuke Shindo * \\ Department of Information Science, Toho University, Miyama, Funabashi, Chiba 274-8510, Japan; \\ yasunori@is.sci.toho-u.ac.jp \\ * Correspondence: 7520001s@st.toho-u.jp
}

Citation: Kimura, Y.; Shindo, K. Asymptotic Behavior of Resolvents of a Convergent Sequence of Convex Functions on Complete Geodesic Spaces. Axioms 2022, 11, 21. https:// doi.org/10.3390/axioms11010021

Academic Editors: Erdal Karapinar, Juan Martínez-Moreno and Inci M. Erhan

Received: 25 November 2021 Accepted: 22 December 2021 Published: 5 January 2022

Publisher's Note: MDPI stays neutral with regard to jurisdictional claims in published maps and institutional affiliations.

Copyright: (C) 2022 by the authors Licensee MDPI, Basel, Switzerland. This article is an open access article distributed under the terms and conditions of the Creative Commons Attribution (CC BY) license (https:// creativecommons.org/licenses/by/ $4.0 /)$.

\begin{abstract}
The asymptotic behavior of resolvents of a proper convex lower semicontinuous function is studied in the various settings of spaces. In this paper, we consider the asymptotic behavior of the resolvents of a sequence of functions defined in a complete geodesic space. To obtain the result, we assume the Mosco convergence of the sets of minimizers of these functions.
\end{abstract}

Keywords: asymptotic behavior; geodesic space; convex function; resolvent

\section{Introduction}

The notion of the resolvent for convex functions is one of the most important subjects in the convex minimization problems. We have proposed various resolvents in many spaces and have studied their properties. Moreover, the asymptotic behavior of resolvents at infinity includes crucial problems in studying the properties of resolvents. There are results on the asymptotic behavior of the resolvent of convex functions at infinity. For example, in a Hilbert space $H$, for a proper lower semicontinuous convex function $f: H \rightarrow]-\infty, \infty]$, a resolvent $J_{f}: X \rightarrow X$ is defined by the following:

$$
J_{f}(x)=\underset{y \in H}{\operatorname{argmin}}\left\{f(y)+\|y-x\|^{2}\right\}
$$

for all $x \in X$. As the asymptotic behavior of this resolvent, the following result is found.

Theorem 1 (See [1]). Let $H$ be a Hilbert space and $f: H \rightarrow]-\infty, \infty]$ a proper lower semicontinuous convex function. For each $x \in X$, if $\left\{J_{\mu_{n} f} x\right\}$ is bounded by some sequence $\left.\left\{\mu_{n}\right\} \subset\right] 0, \infty[$ such that $\mu_{n} \rightarrow \infty$, then $\operatorname{argmin} f \neq \varnothing$ and

$$
\lim _{\lambda \rightarrow \infty} J_{\lambda f} x=P_{\operatorname{argmin} f} x
$$

On the other hand, geodesic spaces are metric spaces which have some convex structures. In geodesic spaces, many types of resolvents are also proposed and studied. A complete CAT(0) space, which is an example of a geodesic space, is a generalization of Hilbert spaces. In this space, the following resolvent is proposed (see [2]). Let $X$ be a complete $\operatorname{CAT}(0)$ space and $f: X \rightarrow]-\infty, \infty]$ a proper lower semicontinuous convex function. We define the resolvent $J_{f}: X \rightarrow X$ of $f$ by the following equation:

$$
J_{f}(x)=\underset{y \in H}{\operatorname{argmin}}\left\{f(y)+d(y, x)^{2}\right\}
$$

for all $x \in X$. For this resolvent, we can also consider asymptotic behavior at infinity and have results similar to Theorem 1 (see [3]). In these cases, a convex function $f$ is fixed. In a Banach space, the convergence of a sequence for resolvents of maximal monotone operators has been considered in many papers. For example, see [4-10]. 
Therefore, we will also consider the convergent sequence of convex functions $\left\{f_{n}\right\}$ and their resolvents. We characterize the convergence of a sequence of convex functions by using the set convergence of minimizers. Mosco convergence is one of the useful notions of set convergence. It is defined in Banach spaces and complete admissible CAT( $\kappa)$ spaces. See [11-13] for more details.

This paper considers the asymptotic behavior of the resolvents of a given convergent sequence of convex functions on a complete $\mathrm{CAT}(0)$ space and a complete admissible CAT(1) space. As a convergence of a sequence of convex functions $\left\{f_{n}\right\}$, we suppose that $\left\{\operatorname{argmin} f_{n}\right\}$, the sequence of sets of minimizers of $f_{n}$, is convergent in the sense of Mosco.

\section{Preliminaries}

Let $X$ be a metric space. For $x, y \in X, c_{x y}:[0, d(x, y)] \rightarrow X$ is called a geodesic with the endpoints $x$ and $y$ if $c_{x y}:[0, d(x, y)] \rightarrow X$ satisfies the following:

$$
\left\{\begin{array}{l}
c_{x y}(0)=x \\
c_{x y}(d(x, y))=y \\
d\left(c_{x y}(u), c_{x y}(v)\right)=|u-v| \text { for } u, v \in[0, d(x, y)] .
\end{array}\right.
$$

We say that $X$ is a uniquely geodesic space if there exists $c_{x y}$ uniquely for each $x, y \in X$. For $x, y \in X$, a geodesic segment $[x, y]$ joining $x$ and $y$ is an image of $c_{x y}$ defined by $[x, y]=$ $c_{x y}([0, d(x, y)])$. A convex combination $z$ between $x$ and $y$ is a point of $[x, y]$ such that $d(x, z)=(1-t) d(x, y)$ and $d(z, y)=t d(x, y)$, and we denote this $z$ by $t x \oplus(1-t) y$. Let $X$ be a uniquely geodesic space and $x_{1}, x_{2}, x_{3} \in X$. A geodesic triangle $\triangle\left(x_{1}, x_{2}, x_{3}\right) \subset X$ with vertices $x_{1}, x_{2}, x_{3}$ is defined by $\triangle\left(x_{1}, x_{2}, x_{3}\right)=\left[x_{1}, x_{2}\right] \cup\left[x_{2}, x_{3}\right] \cup\left[x_{3}, x_{1}\right]$. For a geodesic triangle $\triangle\left(x_{1}, x_{2}, x_{3}\right)$, a comparison triangle $\triangle\left(\bar{x}_{1}, \bar{x}_{2}, \bar{x}_{3}\right) \subset \mathbb{R}^{2}$ is defined as a triangle whose vertices $\bar{x}_{1}, \bar{x}_{2}, \bar{x}_{3}$ satisfy $\left\|\bar{x}_{1}-\bar{x}_{2}\right\|=d\left(x_{1}, x_{2}\right),\left\|\bar{x}_{2}-\bar{x}_{3}\right\|=d\left(x_{2}, x_{3}\right),\left\|\bar{x}_{3}-\bar{x}_{1}\right\|=$ $d\left(x_{3}, x_{1}\right)$. Furthermore, for $p \in\left[x_{i}, x_{j}\right](i, j=1,2,3$ and $i \neq j)$, a comparison point $\bar{p}$ of $p$ is a point on $\left[\bar{x}_{i}, \bar{x}_{j}\right]$ such that $\left\|\bar{p}-\bar{x}_{i}\right\|=d\left(p, x_{i}\right)$. X is called a CAT(0) space if for any geodesic triangle $\triangle\left(x_{1}, x_{2}, x_{3}\right)$, any $p, q \in \triangle\left(x_{1}, x_{2}, x_{3}\right)$, and their comparison points $\bar{p}, \bar{q} \in \bar{\triangle}\left(\bar{x}_{1}, \bar{x}_{2}, \bar{x}_{3}\right)$, the following holds:

$$
d(p, q) \leq\|\bar{p}-\bar{q}\| .
$$

Let $X$ be a geodesic space and $\triangle\left(x_{1}, x_{2}, x_{3}\right)$ a geodesic triangle on $X$. In the same way as above, we define a comparison triangle $\bar{\triangle}\left(\bar{x}_{1}, \bar{x}_{2}, \bar{x}_{3}\right) \subset \mathbb{S}^{2}$. X is called a CAT(1) space if for any geodesic triangle $\triangle\left(x_{1}, x_{2}, x_{3}\right)$ with $d\left(x_{1}, x_{2}\right)+d\left(x_{2}, x_{3}\right)+d\left(x_{3}, x_{1}\right)<2 \pi$, any $p, q \in \triangle\left(x_{1}, x_{2}, x_{3}\right)$, and their comparison points $\bar{p}, \bar{q} \in \bar{\triangle}\left(\bar{x}_{1}, \bar{x}_{2}, \bar{x}_{3}\right)$, it holds that:

$$
d(p, q) \leq d_{\mathbb{S}^{2}}(\bar{p}, \bar{q}) .
$$

An admissible CAT(1) space is a CAT(1) space such that the distance of any two points is smaller than $\pi / 2$. Let $X$ be an admissible CAT(1) space and $\left\{x_{n}\right\}$ a sequence of $X$. The sequence $\left\{x_{n}\right\}$ is said to be spherically bounded if there exists $y \in X$ such that $\sup d\left(x_{n}, y\right)<\pi / 2$ for all $n \in \mathbb{N}$.

We describe the fundamental properties of complete CAT(0) spaces and complete admissible CAT(1) spaces. The following inequalities are called parallelogram laws.

Theorem 2 (See $[3,14]$ ). Let $X$ be a complete CAT $(0)$ space, $x, y, z \in X$, and $t \in[0,1]$. Then,

$$
d(t x \oplus(1-t) y, z)^{2} \leq t d(x, z)^{2}+(1-t) d(y, z)^{2}-t(1-t) d(x, y)^{2} .
$$

Theorem 3 (See [14]). Let X be a complete admissible CAT(1) space, $x, y, z \in X$, and $t \in[0,1]$. Then,

$$
\cos d(t x \oplus(1-t) y, z) \sin d(x, y) \geq \cos d(x, z) \sin t d(x, y)+\cos d(y, z) \sin (1-t) d(x, y) .
$$


In particular, for $t=1 / 2$, it holds that:

$$
\cos d\left(\frac{1}{2} x \oplus \frac{1}{2} y, z\right) \cos \frac{d(x, y)}{2} \geq \frac{1}{2} \cos d(x, z)+\frac{1}{2} \cos d(y, z),
$$

or equivalently, that:

$$
\begin{aligned}
& -\log \cos d\left(\frac{1}{2} x \oplus \frac{1}{2} y, z\right) \\
& \leq \frac{1}{2}(-\log (\cos d(x, z)))+\frac{1}{2}\left(-\log (\cos d(y, z))+\log \cos \frac{d(x, y)}{2} .\right.
\end{aligned}
$$

Let $X$ be a metric space and $\left\{x_{n}\right\}$ a bounded sequence in $X$. For $x \in X$, we assign the following equation:

$$
r\left(x,\left\{x_{n}\right\}\right)=\limsup _{n \rightarrow \infty} d\left(x, x_{n}\right), r\left(\left\{x_{n}\right\}\right)=\inf _{x \in X} r\left(x,\left\{x_{n}\right\}\right) .
$$

Then, if $x \in X$ satisfies $r\left(x,\left\{x_{n}\right\}\right)=r\left(\left\{x_{n}\right\}\right)$, it is called an asymptotic center of $\left\{x_{n}\right\}$. Moreover, if for any subsequence of $\left\{x_{n}\right\}$ its asymptotic center is a unique point $x$, we say that $\left\{x_{n}\right\}$ is $\Delta$-convergent to $x$. Any bounded sequences in complete CAT( 0$)$ space have a $\Delta$-convergent subsequence. Likewise, any spherically bounded sequences in complete admissible CAT $(1)$ have a $\Delta$-convergent subsequence. See [15-17].

Let $X$ be a complete CAT $(0)$ or complete admissible CAT(1) space, and $C$ a closed convex subset of $X$. Then, for $x \in X$, there exists a unique $x_{0} \in C$ such that:

$$
d\left(x_{0}, x\right)=\inf _{y \in C} d(y, x) .
$$

We define $P_{C}: X \rightarrow C$ by the following:

$$
P_{C}(x)=\underset{y \in C}{\operatorname{argmin}} d(y, x) \text {. }
$$

for $x \in X$. This $P_{C}$ is called a metric projection onto $C$ and has the following properties. If $X$ is a complete $\mathrm{CAT}(0)$ space, then

$$
d\left(x, P_{C} x\right)^{2}+d\left(P_{C} x, y\right)^{2} \leq d(x, y)^{2}
$$

for all $y \in C$ and $x \in X$. If $X$ is a complete admissible CAT(1) space, then

$$
\cos d\left(x, P_{C} x\right) \cos d\left(P_{C} x, y\right) \geq \cos d(x, y)
$$

for all $y \in C$ and $x \in X$.

Let $C_{1}, C_{2}, C_{3}, \ldots$ be nonempty closed convex subsets of a complete CAT( 0$)$ or complete admissible CAT(1) space $X$. We define the sets $\mathrm{d}$-Li $C_{n}$ and $\Delta$-Ls $C_{n}$ as follows: $v \in \mathrm{d}-\mathrm{Li} C_{n}$ if and only if there exists $\left\{v_{n}\right\}$ such that $v_{n} \rightarrow v$ and $v_{n} \in C_{n}$ for each $n ; w \in \Delta$-Ls $C_{n}$ if and only if there exists a bounded sequence $\left\{w_{i}\right\}$ such that $w \in A C\left\{w_{i}\right\}$ and $w_{i} \in S_{n_{i}}$ for each $i$. If a closed convex subset $C_{0}$ of $X$ satisfies the following:

$$
\mathrm{d}-\mathrm{Li} C_{n} \subset C_{0} \subset \Delta \text {-Ls } C_{n} \text {, }
$$

we say that $\left\{C_{n}\right\}$ converges to $C_{0}$ in the sense of Mosco and denote $M-\lim _{n \rightarrow \infty} C_{n}=C_{0}$.

\section{Main Results}

Let $f_{1}, f_{2}, f_{3}, \ldots$ be the proper convex lower semicontinuous functions on a CAT(0) or complete admissible CAT(1) space $X$. As the convergence of a sequence of convex functions $\left\{f_{n}\right\}$, we suppose the following conditions:

(a) $\quad \operatorname{M-lim} n \rightarrow \infty \operatorname{argmin} f_{n}=\operatorname{argmin} f$; 
(b) For all $b \in X$, there exists $\left\{b_{n}\right\}$ such that $b_{n} \rightarrow b$ and $\limsup _{n \rightarrow \infty} f_{n}\left(b_{n}\right) \leq f(b)$;

(c) For any subsequence $\left\{f_{n_{i}}\right\}$ of $\left\{f_{n}\right\}$ and a $\Delta$-convergent sequence $\left\{c_{i}\right\}$ whose $\Delta$-limit is $c \in X$, it holds that $f(c) \leq \liminf _{i \rightarrow \infty} f_{n_{i}}\left(c_{i}\right)$.

We consider the asymptotic behavior of a resolvent on CAT(0) space. Let $X$ be a complete $\operatorname{CAT}(0)$ space, $f: X \rightarrow]-\infty, \infty]$ a proper convex lower semicontinuous function, and $x \in X$. We say that a function $\varphi:[0, \infty[\rightarrow \mathbb{R}$ satisfies the condition (A) if the following conditions hold:

- $\varphi$ is increasing;

- $\varphi$ is continuous;

- $\varphi(d(\cdot, x))$ is strictly convex for all $x \in X$;

- $\varphi(t)-k t \rightarrow \infty$ as $t \rightarrow \infty$, for all constants $k \in \mathbb{R}$.

If $\varphi$ satisfies the condition (A), then the function $f(\cdot)+\varphi(d(\cdot, x))$ has a unique minimizer. We define a resolvent $J_{f}$ of $f$ with $\varphi$ by the following equation:

$$
J_{f}(x)=\underset{y \in X}{\operatorname{argmin}}\{f(y)+\varphi(d(y, x))\}
$$

for $x \in X$. For example, $\varphi_{1}(t)=t^{2}$ and $\varphi_{2}(t)=\tanh t \sinh t$ satisfies these conditions. If we define the resolvent with $\varphi_{1}(t)=t^{2}$, it is the resolvent described in the Introduction. For complete CAT $(-1)$ spaces, which are a special case of CAT( 0$)$ spaces, the resolvent with $\varphi_{2}(t)=\tanh t \sinh t$ is defined and studied in [18].

Now we describe the asymptotic behavior of resolvents for a sequence of convex functions satisfying (a), (b), and (c).

Theorem 4. Let $X$ be a complete CAT( 0$)$ space, $\left\{f_{n}\right\}$ a sequence of proper convex lower semicontinuous functions from $X$ to $]-\infty, \infty], f$ a proper convex lower semicontinuous function from $X$ to $]-\infty, \infty]$, and $\left.\left\{\lambda_{n}\right\} \subset\right] 0, \infty\left[\right.$ an increasing sequence diverging to $\infty$. If $\left\{f_{n}\right\}$ and $f$ satisfy the conditions $(a),(b)$, and $(c)$, then for $x \in X$, we have:

$$
\lim _{n \rightarrow \infty} J_{\lambda_{n} f_{n}} x=P_{\operatorname{argmin} f} x .
$$

Proof. Let $x \in X$. We put $x_{n}=J_{\lambda_{n} f_{n}} x$ and $p=P_{\operatorname{argmin} f} x$. Since $p \in \operatorname{argmin} f_{0} \subset$ d-Li argmin $f_{n}$ from the condition (a), there exists $\left\{a_{n}\right\}$ such that $a_{n} \in \operatorname{argmin} f_{n}$ for each $n$ and $a_{n} \rightarrow p$. Since points $a_{n}$ and $x_{n}$ are minimizers of $f_{n}$ and $f_{n}(\cdot)+\varphi(d(\cdot, x))$, respectively, then we have the follwing equation:

$$
\begin{aligned}
f_{n}\left(a_{n}\right)+\frac{1}{\lambda_{n}} \varphi\left(d\left(x_{n}, x\right)\right) & \leq f_{n}\left(x_{n}\right)+\frac{1}{\lambda_{n}} \varphi\left(d\left(x_{n}, x\right)\right) \\
& \leq f_{n}\left(a_{n}\right)+\frac{1}{\lambda_{n}} \varphi\left(d\left(a_{n}, x\right)\right) .
\end{aligned}
$$

Thus, we get $\varphi\left(d\left(x_{n}, x\right)\right) \leq \varphi\left(d\left(a_{n}, x\right)\right)$, which is equivalent to $d\left(x_{n}, x\right) \leq d\left(a_{n}, x\right)$. Since $\left\{a_{n}\right\}$ is a convergent sequence, and $\left\{a_{n}\right\}$ is bounded, this implies that $\left\{x_{n}\right\}$ is also bounded. Take a subsequence $\left\{x_{n_{i}}\right\}$ of $\left\{x_{n}\right\}$ arbitrarily. There exists a $\Delta$-convergent subsequence $\left\{x_{n_{i j}}\right\}$ of $\left\{x_{n_{i}}\right\}$ to some $q \in X$. From the condition (b), there exists $\left\{b_{n}\right\}$ such that $b_{n} \rightarrow p$ and $\lim \sup _{n \rightarrow \infty} f_{n}\left(b_{n}\right) \leq f(p)$. Furthermore, using the condition (c), we get $\liminf _{j \rightarrow \infty} f_{n_{i j}}\left(x_{n_{i j}}\right) \leq f(q)$ as $x_{n_{i j}} \Delta q$. From the definition of the resolvent, we have the following equation:

$$
f_{n_{i j}}\left(x_{n_{i j}}\right)+\frac{1}{\lambda_{n_{i j}}} \varphi\left(d\left(x_{n_{i j}}, x\right)\right) \leq f_{n_{i j}}\left(b_{n_{i j}}\right)+\frac{1}{\lambda_{n_{i j}}} \varphi\left(d\left(b_{n_{i j}}, x\right)\right) .
$$


By the boundedness of $\left\{d\left(x_{n}, x\right)\right\}$ and $\left\{d\left(a_{n}, x\right)\right\}$, letting $j \rightarrow \infty$, we have the following:

$$
f(q) \leq \liminf _{j \rightarrow \infty} f_{n_{i j}}\left(x_{n_{i j}}\right) \leq \liminf _{j \rightarrow \infty} f_{n_{i j}}\left(b_{n_{i j}}\right) \leq \limsup _{j \rightarrow \infty} f_{n_{i j}}\left(b_{n_{i j}}\right) \leq f(p) .
$$

This implies that $q \in \operatorname{argmin} f$. Since $d\left(x_{n_{i j}}, x\right) \leq d\left(a_{n_{i j}}, x\right)$, we let $j \rightarrow \infty$ again and get the following:

$$
d(p, x) \leq d(q, x) \leq \liminf _{j \rightarrow \infty} d\left(x_{n_{i j^{\prime}}}, x\right) \leq \limsup _{j \rightarrow \infty} d\left(x_{n_{i j}}, x\right) \leq \lim _{j \rightarrow \infty} d\left(a_{n_{i j}}, x\right)=d(p, x) .
$$

Hence, we have $q=p$ and $d\left(x_{n_{i j}}, x\right) \rightarrow d(p, x)$. Since $a_{n_{i j}}$ is a minimizer of $f_{n_{i j}}$ and $f_{n_{i j}}$ is convex, we have the following equations:

$$
\begin{aligned}
& f_{n_{i j}}\left(x_{n_{i j}}\right)+\frac{1}{\lambda_{n_{i j}}} \varphi\left(d\left(x_{n_{i j}}, x\right)\right) \\
\leq & f_{n_{i j}}\left(\frac{x_{n_{i j}} \oplus a_{n_{i j}}}{2}\right)+\frac{1}{\lambda_{n_{i j}}} \varphi\left(d\left(\frac{x_{n_{i j}} \oplus a_{n_{i j}}}{2}, x\right)\right) \\
\leq & \frac{f_{n_{i j}}\left(x_{n_{i j}}\right)+f_{n_{i j}}\left(a_{n_{i j}}\right)}{2}+\frac{1}{\lambda_{n_{i j}}} \varphi\left(d\left(\frac{x_{n_{i j}} \oplus a_{n_{i j}}}{2}, x\right)\right) \\
\leq & f_{n_{i j}}\left(x_{n_{i j}}\right)+\frac{1}{\lambda_{n_{i j}}} \varphi\left(d\left(\frac{x_{n_{i j}} \oplus a_{n_{i j}}}{2}, x\right)\right),
\end{aligned}
$$

and hence,

$$
d\left(x_{n_{i j}}, x\right) \leq d\left(\frac{x_{n_{i j}} \oplus a_{n_{i j}}}{2}, x\right) .
$$

From the parallelogram law of CAT(0) space, we get the following:

$$
d\left(x_{n_{i j}}, x\right)^{2} \leq d\left(\frac{x_{n_{i j}} \oplus a_{n_{i j}}}{2}, x\right)^{2} \leq \frac{1}{2} d\left(x_{n_{i j}}, x\right)^{2}+\frac{1}{2} d\left(a_{n_{i j}}, x\right)^{2}-\frac{1}{4} d\left(x_{n_{i j}}, a_{n_{i j}}\right)^{2} .
$$

Since both $\left\{d\left(a_{n_{i j}}, x\right)\right\}$ and $\left\{d\left(x_{n_{i j}}, x\right)\right\}$ are convergent to $d(p, x)$, we have:

$$
d\left(x_{n_{i j}}, a_{n_{i j}}\right)^{2} \leq 2\left(d\left(a_{n_{i j}}, x\right)^{2}-d\left(x_{n_{i j}}, x\right)^{2}\right) \rightarrow 0
$$

which implies that $x_{n_{i j}} \rightarrow p$. Then, any subsequence $\left\{x_{n_{i}}\right\}$ of $\left\{x_{n}\right\}$ has a convergent subsequence $\left\{x_{n_{i j}}\right\}$, which tends to $p$. From these facts, we get a desired result.

From this theorem, we have the following corollaries. Suppose $f_{n}=f$ for all $n \in \mathbb{N}$. Then $\left\{f_{n}\right\}$ obviously satisfies the conditions (a), (b), and (c).

Corollary 1. Let $X$ be a complete $\mathrm{CAT}(0)$ space, $f$ a proper convex lower semicontinuous function from $X$ to $]-\infty, \infty]$, and $\varphi:[0, \infty[\rightarrow \mathbb{R}$ a function satisfying the condition (A). For a positive real number $\lambda$, define $J_{\lambda f}: X \rightarrow X$ by the following equation:

$$
J_{\lambda f}(a)=\underset{y \in X}{\operatorname{argmin}}\{\lambda f(y)+\varphi(d(y, a))\}
$$

for $a \in X$. Then, for each $x \in X$,

$$
\lim _{\lambda \rightarrow \infty} J_{\lambda f} x=P_{\operatorname{argmin} f} x .
$$

Let $\left\{C_{n}\right\}$ be a sequence of nonempty closed convex subsets which converges to $C$ in the sense of Mosco. If $\left\{f_{n}\right\}=\left\{i_{C_{n}}\right\}$ and $f=i_{C}$, then $\operatorname{argmin} f_{n}=C_{n}$ and $\operatorname{argmin} f=C$, 
where $i_{C}$ is the indicator function of $C$. Since $\left\{C_{n}\right\}$ converges to $C,\left\{i_{C_{n}}\right\}$ and $i_{C}$ satisfy the condition (a). They also satisfy the conditions (b) and (c).

Corollary 2. Let $X$ be a complete $\mathrm{CAT}(0)$ space, $\left\{C_{n}\right\}$ a sequence of nonempty closed convex subsets of $X$, and $C$ a nonempty closed convex subset of $X$. If $\left\{C_{n}\right\}$ converges to $C$ in the sense of Mosco, then for each $x \in X$,

$$
\lim _{n \rightarrow \infty} P_{C_{n}} x=P_{C} x
$$

Similarly, we consider asymptotic behavior of a resolvent on CAT(1) space. Let $X$ be a complete admissible CAT(1) space. We say $\varphi:[0, \pi / 2[\rightarrow \mathbb{R}$ satisfies the condition (B) if the following hold:

- $\varphi$ is increasing;

- $\varphi$ is continuous;

- $\varphi(d(\cdot, x))$ is strictly convex for all $x \in X$;

- $\varphi(t) \rightarrow \infty$ as $t \rightarrow \pi / 2$.

Then, the set $\operatorname{argmin}_{y \in X}\{f(y)+\varphi(d(y, x))\}$ is a singleton for all $x \in X$, and we define $J_{f}$ in a similar way. For example, $\varphi_{3}(t)=\tan t \sin t$ and $\varphi_{4}(t)=-\log \cos t$ satisfy the conditions above. On complete admissible CAT(1) spaces, resolvents by using these functions are defined and their properties are studied in $[19,20]$.

We consider that the asymptotic behavior of resolvents for a sequence of convex functions satisfies (a), (b), and (c).

Theorem 5. Let $X$ be a complete admissible CAT(1) space, $\left\{f_{n}\right\}$ a sequence of proper convex lower semicontinuous functions from $X$ to $]-\infty, \infty], f$ a proper convex lower semicontinuous function from $X$ to $]-\infty, \infty]$, and $\left.\left\{\lambda_{n}\right\} \subset\right] 0, \infty\left[\right.$ an increasing sequence diverging to $\infty$. If $\left\{f_{n}\right\}$ and $f$ satisfy the conditions (a), (b), and (c), then for $x \in X$,

$$
\lim _{n \rightarrow \infty} J_{\lambda_{n} f_{n}} x=P_{\operatorname{argmin} f} x .
$$

Proof. In the same way as the proof of Theorem 5, if we take $\left\{x_{n_{i j}}\right\}$ with the same procedure, it hold that $d\left(x_{n_{i j}}, x\right) \rightarrow d(p, x)$ and

$$
d\left(x_{n_{i j}}, x\right) \leq d\left(\frac{x_{n_{i j}} \oplus a_{n_{i j}}}{2}, x\right) .
$$

From the parallelogram law of CAT(1) space, we get the following equations:

$$
\begin{aligned}
-\log \left(\cos d\left(x_{n_{i j}}, x\right)\right) \leq & -\log \left(\cos d\left(\frac{x_{n_{i j}} \oplus a_{n_{i j}}}{2}, x\right)\right) \\
\leq & \frac{1}{2}\left(-\log \left(\cos d\left(x_{n_{i j}}, x\right)\right)\right)+\frac{1}{2}\left(-\log \left(\cos d\left(a_{n_{i j}}, x\right)\right)\right) \\
& -\frac{1}{4}\left(-\log \left(\cos d\left(x_{n_{i j}}, a_{n_{i j}}\right)\right)\right) .
\end{aligned}
$$

Then, we have

$$
-\log \left(\cos d\left(x_{n_{i j}}, a_{n_{i j}}\right)\right) \leq 2\left(-\log \left(\cos d\left(x_{n_{i j}}, x\right)\right)-\log \left(\cos d\left(a_{n_{i j}}, x\right)\right)\right) .
$$

This implies that $x_{n_{i j}} \rightarrow p$, and we get $J_{\lambda_{n} f_{n}} x \rightarrow P_{\operatorname{argmin} f} x$.

As well as for the case of CAT(0) spaces, we obtain the following corollaries in CAT(1) spaces. 
Corollary 3. Let $X$ be a complete admissible CAT(1) space, $f$ a proper convex lower semicontinuous function from $X$ to $]-\infty, \infty]$, and $\varphi:[0, \pi / 2[\rightarrow \mathbb{R}$ a function satisfying the condition (B). For $a$ positive real number $\lambda$, define $J_{\lambda f}: X \rightarrow X$ by the following equation:

$$
J_{\lambda f}(a)=\underset{y \in X}{\operatorname{argmin}}\{\lambda f(y)+\varphi(d(y, a))\}
$$

for $a \in X$. Then, for each $x \in X$, we have:

$$
\lim _{\lambda \rightarrow \infty} J_{\lambda f} x=P_{\operatorname{argmin} f} x
$$

Corollary 4. Let $X$ be a complete admissible CAT(1) space, $\left\{C_{n}\right\}$ a sequence of nonempty closed convex subsets of $X$, and $C$ a nonempty closed convex subset of $X$. If $\left\{C_{n}\right\}$ converges to $C$ in the sense of Mosco, then for each $x \in X$, we have:

$$
\lim _{n \rightarrow \infty} P_{C_{n}} x=P_{C} x
$$

\section{Applications to Hilbert Spaces}

Finally, we consider the applications of our results to the case of a Hilbert space. Because the class of complete CAT(0) spaces includes that of Hilbert spaces, we can get some results in Hilbert spaces directly. The definitions of conditions for functions $\varphi$ and $\left\{f_{n}\right\}$ are applied to those in CAT(0) spaces.

Theorem 6. Let $H$ be a Hilbert space, $\left\{f_{n}\right\}$ a sequence of proper convex lower semicontinuous functions from $X$ to $]-\infty, \infty], f$ a proper convex lower semicontinuous function from $X$ to $]-\infty, \infty]$, and $\left.\left\{\lambda_{n}\right\} \subset\right] 0, \infty\left[\right.$ an increasing sequence diverging to $\infty$. Suppose $\left\{f_{n}\right\}$ and $f$ satisfy the conditions (a), (b), and (c), and $\varphi:\left[0, \infty\left[\rightarrow \mathbb{R}\right.\right.$ satisfies the condition $(A)$. Define $J_{\lambda_{n} f_{n}}: X \rightarrow X$ by the following:

$$
J_{\lambda_{n} f_{n}}(a)=\underset{y \in X}{\operatorname{argmin}}\left\{\lambda_{n} f_{n}(y)+\varphi(\|y-a\|)\right\}
$$

for $a \in X$. Then, for each $x \in X$

$$
\lim _{n \rightarrow \infty} J_{\lambda_{n} f_{n}} x=P_{\operatorname{argmin} f} x
$$

Using this result, we can get following famous theorems. First, if we consider the case that a convex function is fixed and $\varphi(t)=t^{2}$, we can get Theorem 1 . Next, considering the case that convex functions are the indicator functions of some convex sets, we obtain the following theorem.

Theorem 7 (See [21]). Let $H$ be a Hilbert space, $\left\{C_{n}\right\}$ a sequence of nonempty closed convex subsets of $X$, and $C$ a nonempty closed convex subset of $X$. If $\left\{C_{n}\right\}$ converges to $C$ in the sense of Mosco, then for each $x \in X$,

$$
\lim _{n \rightarrow \infty} P_{C_{n}} x=P_{C} x
$$

In conclusion, we summarize the results in this paper. For a given sequence $\left\{f_{n}\right\}$ of proper lower semicontinuous functions converging to $f$ in the sense of the conditions (a), (b), and (c), we consider the corresponding sequence of resolvents $\left\{J_{\lambda_{n} f_{n}}\right\}$ with a positive real sequence $\left\{\lambda_{n}\right\}$ diverging to $\infty$. The main results imply the pointwise convergence of this sequence to the metric projection onto $\operatorname{argmin} f$ in the setting of a CAT(0) and a CAT(1) space, respectively. We can apply them to the asymptotic behavior of the resolvent for a single function at $\infty$, and a convergence theorem for a sequence of metric projections.

Author Contributions: The authors (Y.K. and K.S.) have contributed to this work on an equal basis. All authors have read and agreed to the published version of the manuscript. 
Funding: This work was partially supported by JSPS KAKENHI, Grant Number JP21K03316.

Conflicts of Interest: The authors declare no conflict of interest.

\section{References}

1. Takahashi, W. Nonlinear Functional Analysis Fixed Point Theory and Its Applications; Yokohama Publishers: Yokohama, Japan, 2000.

2. Mayer, U.F. Gradeient flows on nonpositively curved metric space and harmonic maps. Commun. Anal. Geom. 1998, 6, 45-56. [CrossRef]

3. Bačák, M. Convex Analysis and Optimaization in Hadamard Spaces; De Gruyter: Berlin, Germany, 2014.

4. Kamimura, S.; Takahashi, W. Approximating solutions of maximal monotone operators in Hilbert spaces. J. Approx. Theory 2000, 106, 226-240. [CrossRef]

5. Kamimura, S.; Takahashi, W. Weak and strong convergence of solutions to accretive operator inclusions and applications. Set-Valued Anal. 2000, 8, 361-374. [CrossRef]

6. Kamimura, S.; Takahashi, W. Strong convergence of a proximal-type algorithm in a Banach space. SIAM J. Optim. 2002, 13, 938-945. [CrossRef]

7. Kido, K. Strong convergence of resolvents of monotone operators in Banach spaces. Proc. Am. Math. Soc. 1988, 103, 755-758. [CrossRef]

8. Otero, R.G.; Svaiter, B.F. A strongly convergent hybrid proximal method in Banach spaces. J. Math. Anal. Appl. 2004, $289,700-711$. [CrossRef]

9. Reich, S. Constructive techniques for accretive and monotone operators. In Applied Nonlinear Analysis (Proceedings Third International Conference, University Texas, Arlington, TX, 1978); Academic Press: New York, NY, USA, 1979; pp. 335-345.

10. Reich S. Strong convergence theorems for resolvents of accretive operators in Banach spaces. J. Math. Anal. Appl. 1980 75, $287-292$. [CrossRef]

11. Mosco, U. Convergence of convex sets and of solutions of variational inequalities. Adv. Math. 1969, 3, 510-585. [CrossRef]

12. Beer, G. Topologies on Closed and Closed Convex Sets; Kluwer Academic Publishers Group: Dordrecht, The Netherlands, 1993.

13. Kimura, Y.; Satô, K. Convergence of subsets of a complete geodesic spaces with curvature bounded above. Nonlinear Anal. 2012, 75, 5079-5085. [CrossRef]

14. Bridson, M.R.; Haefliger, A. Metric spaces of non-positive curvature. In Fundamental Principles of Mathematical Sciences; Springer: Berlin/Heidelberg, Germany, 1999; Volume 319.

15. Lim, T.C. Remarks on some fixed point theorems. Proc. Am. Math. Soc. 1976, 60, 179-182. [CrossRef]

16. Kirk, W.A.; Panyanak, B. A concept of convergence in geodesic spaces. Nonlinear Anal. 2008, 68, 3689-3696. [CrossRef]

17. Espínola, R.; Fernández-León, A. CAT(k)-spaces, weak convergence and fixed points. J. Math. Anal. Appl. 2009, 353, 410-427. [CrossRef]

18. Kajimura, T.; Kimura, Y. Resolvents of convex functions in complete geodesic metric spaces with negative curvature. J. Fixed Point Theory Appl. 2019, 21, 32. [CrossRef]

19. Kimura, Y.; Kohsaka, F. Spherical nonspreadingness of resolvents of convex functions in geodesic space. J. Fixed Point Theory Appl. 2016, 18, 93-115. [CrossRef]

20. Kajimura, T.; Kimura, Y. A New Resolvent for Convex Functions in Complete Geodesic Spaces. RIMS Kôkyûroku 2019, 2112, 141-147.

21. Tsukada, M. Convergence of best approximations in a smooth Banach space. J. Approx. Theory 1984, 40, 301-309. [CrossRef] 